\title{
STRENGTH EVALUATION IN TURBO MACHINERY BLADE DISK ASSEMBLY AT CONSTANT SPEED
}

\author{
K. Kumar ${ }^{1}$, S. L. Ajit Prasad ${ }^{1}$ and Shivarudraiah ${ }^{2}$ \\ ${ }^{1}$ Mechanical Engineering, \\ PES College of Engineering, Mandya, India \\ Email: kumar.triveni@gmail.com \\ ${ }^{2}$ Mechanical Engineering, UVCE Bangalore, India.
}

\begin{abstract}
In the present work, 3D finite element analyses (FEA) of a low pressure (LP) steam turbine bladed disk assembly are carried out at constant speed loading condition. The prime objective of this work is to optimize the geometry of the bladed disk root with the aid of the Peterson's stress concentration factor (SCF) charts available in the literature. Secondly, design rules are developed for the structural integrity of the blades and disk considering a safety factor for material, manufacturing and temperature uncertainties. These design rules are in turn used as design checks with the aid of finite element analysis results. Special investigations were performed based on Neuber formulae for reducing the local peak stresses at the blade and disk root fillet using linear analysis to identify the equivalent non linear stress values by the strain energy distribution method for estimating the minimum number of cycles required for the onset of crack initiation.
\end{abstract}

Keywords: Stress concentration factor, Neuber rule, steam turbine blades, peak stresses, strain energy.

\section{INTRODUCTION}

The importance of power generation through thermal and nuclear plants is very much felt due to uneven fluctuations in the monsoon, which has made power generation and supply inconsistent. With the ever-increasing demand for power due to increased industrial activities, it has become imperative to generate power economically and consistently. One such attempt has resulted in the increased use of steam turbines for power generation. In a steam turbine, a number of blades are used for energy transfer (Kearton, 1988; Mazur et al., 2009). These blades are subjected to centrifugal force and are critically important parts which affect the functioning of a turbine. Bladed disks are flexible elements in high speed rotating machinery. Due to rotation, the blade root gets tightened in the disk slot and transmits the centrifugal load. The mating contact may be just two or may increase to six or more for low-pressure blades. While the average stress in the mating surface is fully elastic and well below yield, the peak stress at singularities in the groove shape can reach yield values and reach into the local plastic region (Bloch, 1982). Last stage LP turbine blades are the most severely stressed blades in the system. Usually these are the limiting cases of blade design allowing the peak stress to reach yield or just below yield conditions. Failure can occur with crack initiation at the stress raiser location and then propagation. 
Optimization methods have become available to optimize the shape and minimize the peak stress values so as to improve the structural integrity of the bladed disk. Because the problem is highly nonlinear due to centrifugal stiffening and spin softening, considerable time is required to reach the optimized root using Peterson's stress concentration chart (Pilkey, 1997).There is a vast literature on linear-elastic stress concentration factors $K_{t}$, which depend solely on the specimen/notch geometry and on the type of loading. However, in the presence of plasticity at the notch root, the actual stress concentration factor $K_{\sigma}$ is found to be smaller than the tabulated $K_{t}$, mainly due to stress redistribution at the yielding zone. In turn, the strain concentration factor $K_{\varepsilon}$ at the notch root, which strongly affects the fatigue life predicted by the strain based approach $(\varepsilon N)$ method, can be much larger than $K_{t}$ (Kim, 1998). In many cases, these models may provide reasonable estimates of the maximum stresses and strains at the notch root; however, the differences among the fatigue life predictions by each rule can be unacceptably large. In addition, these methods do not account for the geometrical changes at the notch root under large displacements, leading to further errors.

In light of the above, in the present paper knowledge based engineering is applied to develop design rules for the structural integrity of blades at the design stage. These rules are used as design checks in finite element analysis; Neuber's technique of using linear analysis to predict non-linear stress/strain values in the component is effectively utilized to solve this highly non-linear problem based on the strain energy redistribution method. A sensitivity study on the shape variables of the root geometry is done to finalize the root dimensions based on Peterson's charts, to ensure manufacturing feasibility and structural integrity. The main objective of the present study is to carry out structural analyses of the LP stage blade disk sector for its strength evaluation. This includes:

1) Development of a design rule for turbo machinery blades that ensure the structural requirements;

2) Effective utilization of the design rule to meet the structural requirements on a par with the finite element results as a design check;

3) Modification of the blade root to reduce the stress concentration at the root and modify the disk corresponding to the modified blade root dimension as per Peterson's SCF charts.

\section{MATERIALS AND METHODS}

\section{Blade Type}

Following the steam path through a turbine the environment for the energy converging blades varies strongly and, as a consequence, so do the mechanical requirements. These requirements have a strong influence on the choice of material and design with respect to temperature, wetness and cleanliness of medium, acting forces and other factors as, e.g., hardenability and oxidation. Different blade families exist which can be categorized according to their use in the primary three turbine modules as high, intermediate and low-pressure blades (HP, IP and LP). Generally the LP stage blades are shrouded or laced, because they have to resist huge centrifugal forces and bending produced by the steam loads. Figure 1 shows the geometry of the LP blade. 


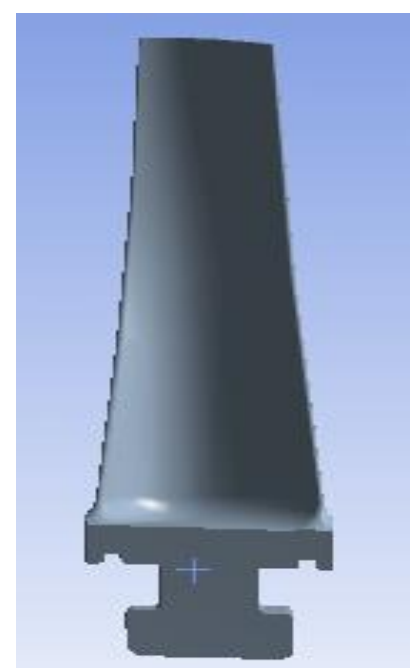

Figure 1: Simple 'T' root LP Blade

\section{Design Development}

Design rule margins are necessary because of the uncertainties present in material, manufacturing, assembly and on site operating conditions. As per API standards the blades should prove its integrity at design speed and over-speed conditions. Based on knowledge base engineering in the present work, a $121 \%$ margin is considered for $11 \%$ over speed due to full fuel throw-off condition. An additional margin of $15 \%$ is considered for manufacturing and thermal uncertainties. Therefore the allowable stress in the component at design speed may be written as

$$
\sigma_{\text {available }}=\frac{\sigma_{\min }}{(\text { over speed })^{2} \times \text { additional FOS }}
$$

The speed load contribution is high in rotating machinery and the centrifugal force varies as $F_{c}=m \omega^{2} r$. Care should be exercised for the speed load, which varies as a square function. With this assumption at design speed, the factor of safety (FOS) = $(1.212 \times 1.15)=1.68$. The allowable FOS at different speeds is as shown in Table 1 . This argument shows that the material has a $15 \%$ additional margin at $121 \%$ over speed condition. The allowable average stress limit at an average stage temperature of $75^{\circ} \mathrm{C}$ for the blade material with a minimum yield of $585 \mathrm{MPa}$ is $349 \mathrm{MPa}$.

Table 1: Factor of safety limits

\begin{tabular}{lc}
\hline Speed & Allowable FOS \\
\hline $100 \%$ & 1.68 \\
$110 \%$ & 1.38 \\
$121 \%$ & 1.15 \\
\hline
\end{tabular}




\section{Section Stress}

It is seen that low-pressure blades are highly subject to centrifugal and thermomechanical loads. Since the centrifugal load dominates the thermal loads for every mission cycle, emphasis is paid on addressing mechanical loads. In a bladed disk assembly the blade happens to be the weaker section compared to disk. Replacing blades under failure is much cheaper than replacing disks. Average section stresses attained at minimum cross sections at operating and at over-speed conditions should be within the allowable design limits following the design rule both for blade and disk. The design rule is that the average section stress in the disk groove should have a greater margin compared to the blades, based on the 'weak link' analogy. The following are the critical zones identified in a bladed disk assembly. The average section stress in the airfoil should be less than the allowable stress limits at design speed.

a) The average section stress at blade root neck and at minimum cross-section of disk should be within the allowable design limits (Kubiak Sz , 2009).

b) The average section stress at minimum cross section of disk should be $10 \%$ less than the blade root neck average stress, based on the 'weak link' analogy.

c) The average section stress in the disk groove considering loading and unloading characteristics at over-speed conditions should have a margin of 1.18 at $0.2 \%$ proof stress of the material. The behaviour constraints to evaluate the over-speed and burst margin may be as follows:

d) Behaviour Constraints

Allowable hoop and von Mises stress at the bore is $95 \%$ of $0.2 \%$ proof stress. Allowable hoop stress at the web is $85 \%$ of $0.2 \%$ proof stress.

Allowable radial and von Mises stress at the web is $80 \%$ of $0.2 \%$ proof stress. Allowable hoop and von Mises stress at the rim is $67 \%$ of $0.2 \%$ proof stress. Allowable radial growth < specified tolerance of $3 \mathrm{~mm}$.

Allowable axial growth < specified tolerance of $1 \mathrm{~mm}$.

Allowable AWMHS $<72 \%$ of $0.2 \%$ proof stress at peak temperature in the disk. Allowable AWMHS < $64 \%$ of UTS at peak temperature in the disk.

Disk burst speed $\geq 125 \%$ of maximum allowable steady state speed of $6000 \mathrm{rpm}$.

Disk over-speed $\geq 118 \%$ of maximum allowable steady state speed.

In addition to the standard behaviour constraints on stresses, deflections, eigenvalues and buckling load factors, there are constraints related to the area weighted mean hoop stress (AWMHS) and the area weighted mean radial stress (AWMRS) (Richter, 2003). These constraints are specific to rotor design in the aero-engine industry and therefore not readily available from a general-purpose program like ANSYS and hence many non-standard constraints have been incorporated into design rules for the effective evaluation of rotor components. 


\section{Fatigue Life Approach}

Steam turbine blades are constant speed machines which rarely undergo one-over-speed for every start up and shut down cycle. The blade loading pattern is as shown in Figure 2.

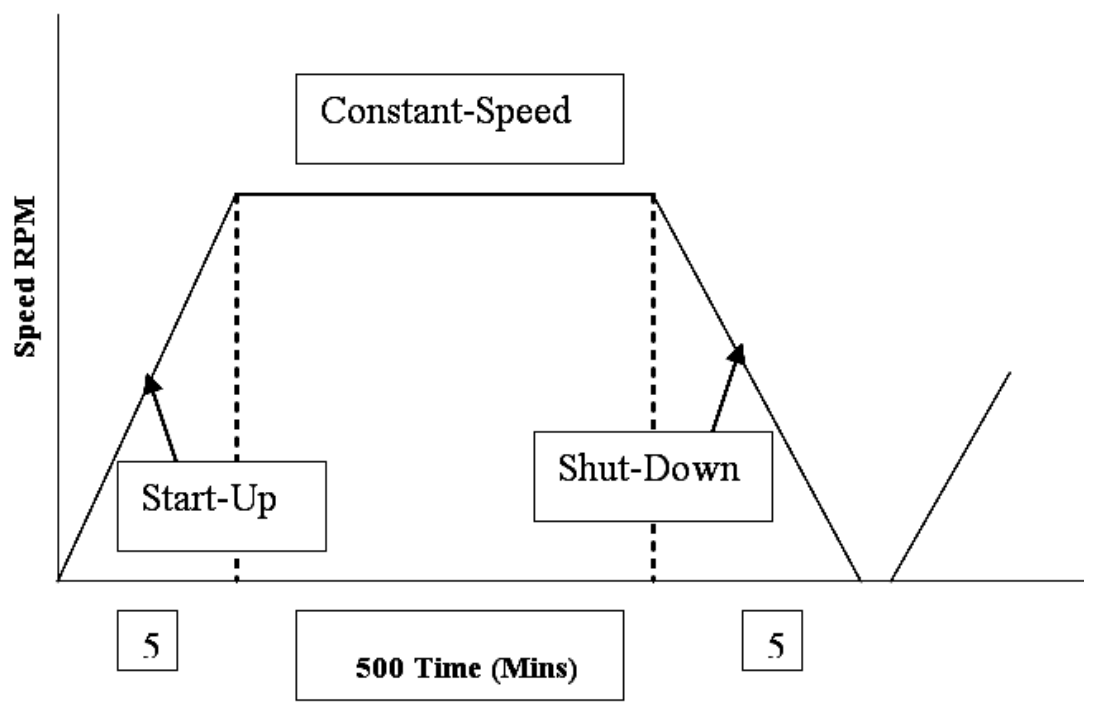

Figure 2: Blade Loading Pattern

In the present work it is desired to achieve a minimum of 2500 cycles in both blade and disk. Often the blade root fillets and disk groove fillets undergo their highest peak stress/strain for every cyclic loading (start up cycle). Therefore some amount of plastic strain gets accumulated each cycle (Mazur, 2008). The total strain accumulated is the sum of the elastic and plastic strain. A knock down factor of 2 is considered for fatigue modifying factors like size factors, surface factors, reliability factor and temperature factor. For better design with these uncertainties the actual life at the design stage is 5000 cycles. Lifing is generally done with the classical approach, considering all the fatigue strength modifying factors in the stress based and strain based approach The $\varepsilon N$ material curve data for a given material at operating temperature is utilized for lifing the blade and disk based on the local strain approach. For the stress based approach, the total stress/strain is estimated using Neuber's rule. The nonlinear stress and $S-N$ material curve for notched specimens at operating temperature is used to achieve the minimum start up shut down cycles. In the present work one such effort is made to reduce the peak stresses at blade root fillet to improve the life of the blade disk assembly.

The stress based theory has been recognized as much slower in modelling the crack initiation phase; the most promising approach seems to be based on the strain approach. Even if the crack is not initiated in the model, the life of the component before the crack initiates is of concern. The basic premise is the local fatigue response of the material at the critical point, i.e., at the site of crack initiation. It is analogous to the fatigue response of a component subjected to cyclic stresses, to properly perform the local cyclic stress strain history at the critical point. The structure must be determined either by analytical or experimental means. In the light of the above cyclic symmetry, pre-stress static analysis with different load step procedure as per the blade loading 
pattern is conducted using finite element analysis. The available stress/strain in the component from the analysis and material fatigue curve calculations is then conducted.

\section{RESULTS AND DISCUSSION}

\section{Finite Element Model and Analysis}

In the present analysis a cyclic symmetry sector of a bladed disk assembly of the LP stage is considered. The geometry of one blade with disk sector is modelled using the commercially available modelling software, Pro-E. The finite element model of the LP sector stage bladed disk is as shown in Figure 3. For the present analysis, SOLID45 element is considered to generate the finite element model. No degenerated tetrahedral elements exist in the mesh, as the de-generated tetrahedral elements are not good for accurate stress calculations. Surface to surface contact pairs were created between the blade and disk mating surfaces. A friction co-efficient of 0.1 is assumed at the butting faces of blade and disk. A matching node pattern is maintained at the blade root and disk hook pressure faces (in order to avoid any ambiguities in stress values due to contact elements), where the load transfer between blade and disk takes place. Cyclic boundary conditions are applied on both the partition surfaces. The blade and disk both are assumed to be made of the same material with a yield stress of $585 \mathrm{MPa}$, Young's modulus $210 \mathrm{GPa}$, density $7900 \mathrm{~kg} / \mathrm{m}^{3}$ and Poisson's ratio 0.3 .
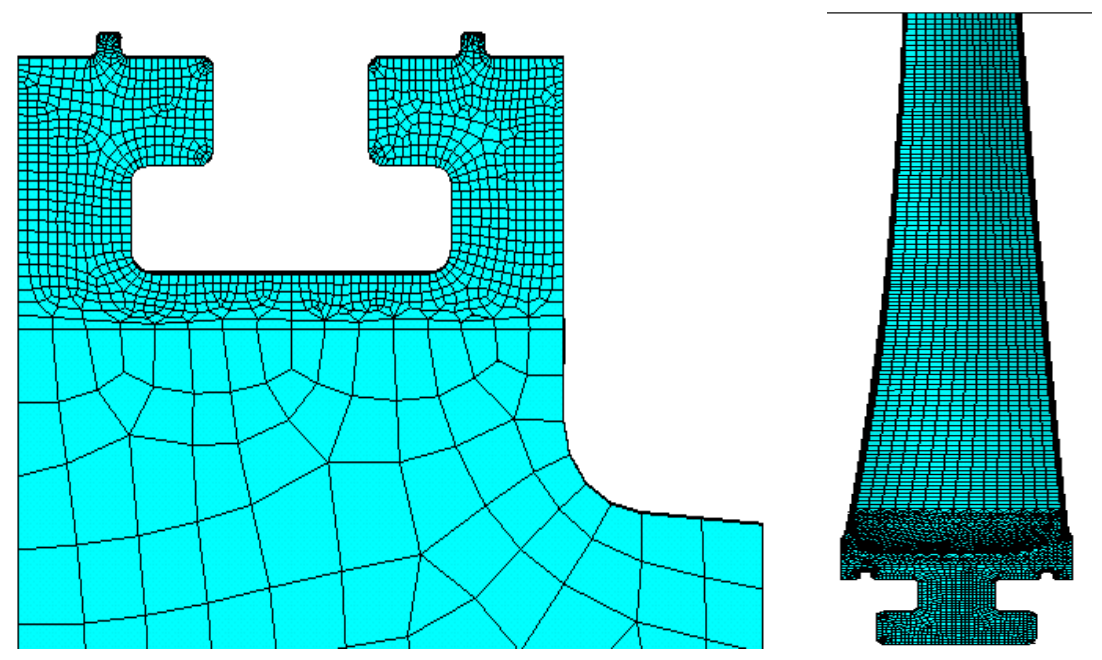

Figure 3: FE Model of Blade Disk Sector

The peak von Mises stress computed in the blade root fillet and disk hook fillet are shown in Figure 4 and Figure 5. The stresses are much higher than the yield stress (585 MPa) of the material. Hence, the use of linear analysis was alarming. However, traditional nonlinear FEA analyses simulating the local material plasticity are still very resource intensive, yet fatigue and life endurance simulations commonly need stress and strain results for various load levels, making such an analysis expensive. In order to reduce the number of nonlinear simulation results, approximation techniques based on Neuber's formula, which estimate the plastic stress-strain state from linear analysis runs, were utilized. With the application of FEA, notch concentration factors are being inherently considered. The general Neuber's procedure of extrapolating linear stresses into the plastic material region can thus be applied to arbitrary geometries. 
The discussions for comparison and design decisions are based on stresses after Neuber's procedure. The baseline geometry shows a peak stress of $1904 \mathrm{MPa}$ in the blade root fillet, it lead to very poor LCF cycles of 2400 cycles, calculated based on the strain approach from Neuber's formula. Also the knowledge based design criteria (established based on experience and experimental tests) limits the peak stress to 1200 $\mathrm{MPa}$ from the linear finite element stress analysis, for a component life of 5000 startup/shut-down cycles. The average section stress at minimum disk area is $210 \mathrm{MPa}$, which clearly satisfies the design rule as discussed earlier. Since the baseline model shows a peak stress of $1904 \mathrm{MPa}$ from the linear stress analysis, optimization of the blade root is required to bring down the peak stress. Though the average section stress in blade and disk critical locations experience a margin sufficient to satisfy the design rule: the design rule for average section stresses as discussed earlier in airfoil, blade root and disk groove minimum cross-section is well within the allowable design limits of $349 \mathrm{MPa}$ at design speed. The necessary design margins at over speed conditions with radial growth at an average stage temperature of $70^{\circ} \mathrm{C}$ as per the design rule is satisfied. The local peak stress/strain at the blade root fillet was not sufficient to achieve the minimum desired 5000 cycle. The radial growth of the blade at operating conditions is well within the clearance of $3 \mathrm{~mm}$ between blade tip and casing. This indicates that there is no rubbing of the blade with the casing even at over speed conditions.
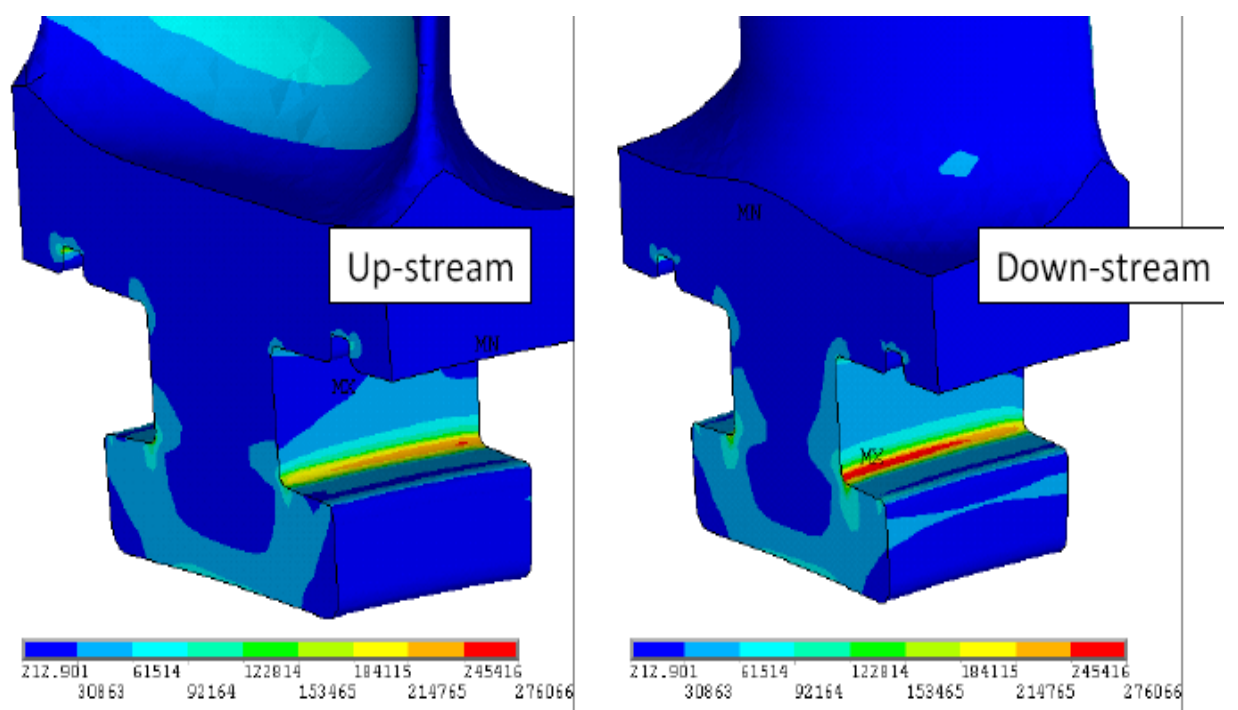

Peak stress and average stress are $1904 \mathrm{MPa}$ at blade root and $250 \mathrm{MPa}$ at blade neck

Figure 4: Von Mises stress at $6000 \mathrm{rpm}$ in elastic domain. 


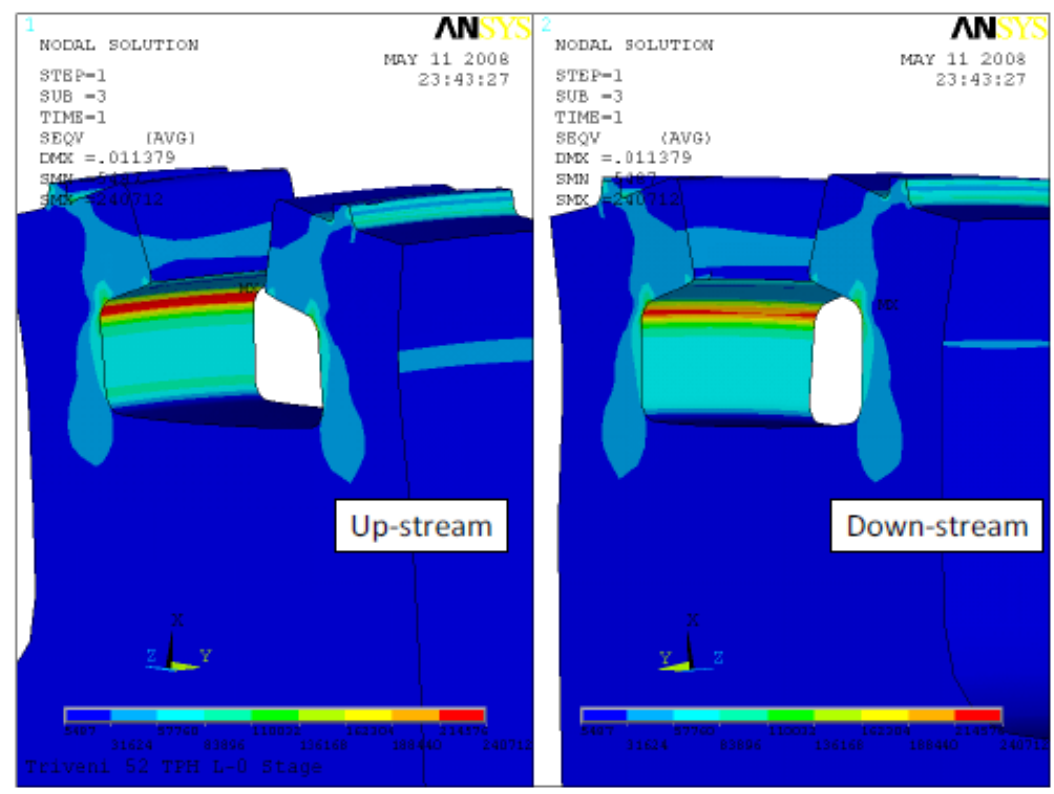

Peak stress of $1660 \mathrm{MPa}$ at blade root

Figure 5: Von Mises stress at $6000 \mathrm{rpm}$ in elastic domain of disk

\section{Optimization}

Experience shows that in order to improve the LCF life of a bladed disk assembly, a corrective measure usually involves a design or geometry modification of the blade root section where the peak stress occurs due to the change in geometry. By studying stress concentration factors at the blade root, much can be learned about how to produce designs that are superior from the stand-point of resistance to repeated loads, and how to approximately evaluate the influence of various geometric features. The chart from Peterson's book (Pilkey, 1997) was used as an edge finder to reduce the SCF. The blade root geometry dimension was modified to have a theoretical SCF of 6 whereas the baseline geometry has a SCF of 10.4. The modified geometric model is analysed using ANSYS to study the sensitivity of the modified geometry. The modification of the blade root is shown in Table 2 and the different parameters in Table 2 are illustrated in Figure 6.

Table 2. Root Terminology

\begin{tabular}{lrrrrrrr}
\hline \multicolumn{10}{c}{ Dimensional details of blade root } \\
\hline d & D & m & r & r/d & L & W \\
\hline Baseline root & 22 & 40 & 13 & 1 & 0.04 & 6 & 65 \\
Modified root & 22 & 45 & 14 & 2.2 & 0.1 & 7 & 77 \\
\hline
\end{tabular}




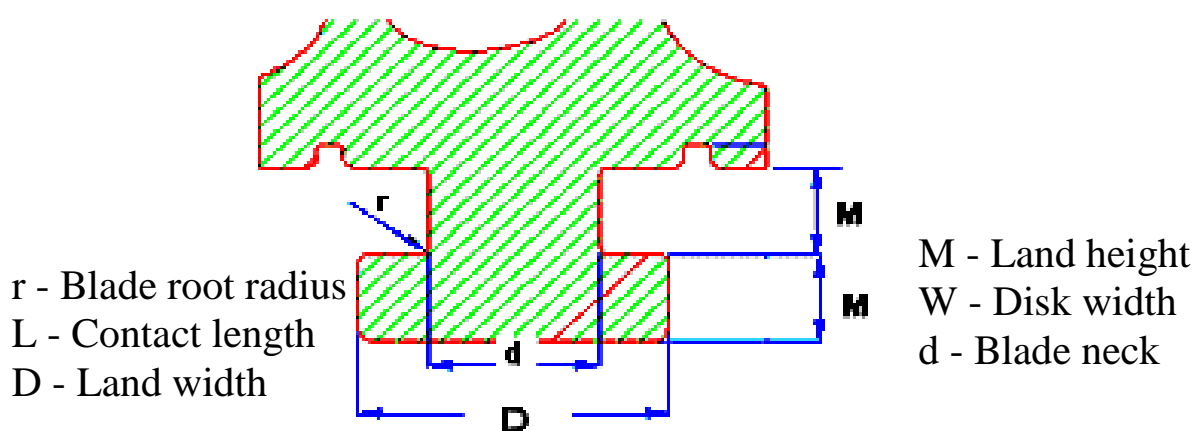

Figure 6: Root Terminology

The peak stress of the modified blade root geometry is $1153 \mathrm{MPa}$, as shown in Figure 7, which is smaller than the previous peak stress of $1904 \mathrm{MPa}$ at the blade root fillet. In a similar manner, the peak stress in the disk hook decreasedto $1102 \mathrm{MPa}$ from $1660 \mathrm{MPa}$, which is shown in Figure 8. From the gross yielding point of view, the average stress in the blade neck was $242 \mathrm{MPa}$ with FOS of 1.68 at minimum yield at room temperature. The same exercise was repeated for $121 \%$ over speed condition at $7200 \mathrm{rpm}$. At this over speed condition, the induced stresses were under the satisfactory limit for the required LCF life. The modified blade root does not differ from average stress point of view, over-speed margin or radial growth, since not much change in the blade and disk is noticed, except for blade root and disk groove fillet radius which alters the peak stresses necessary for LCF.
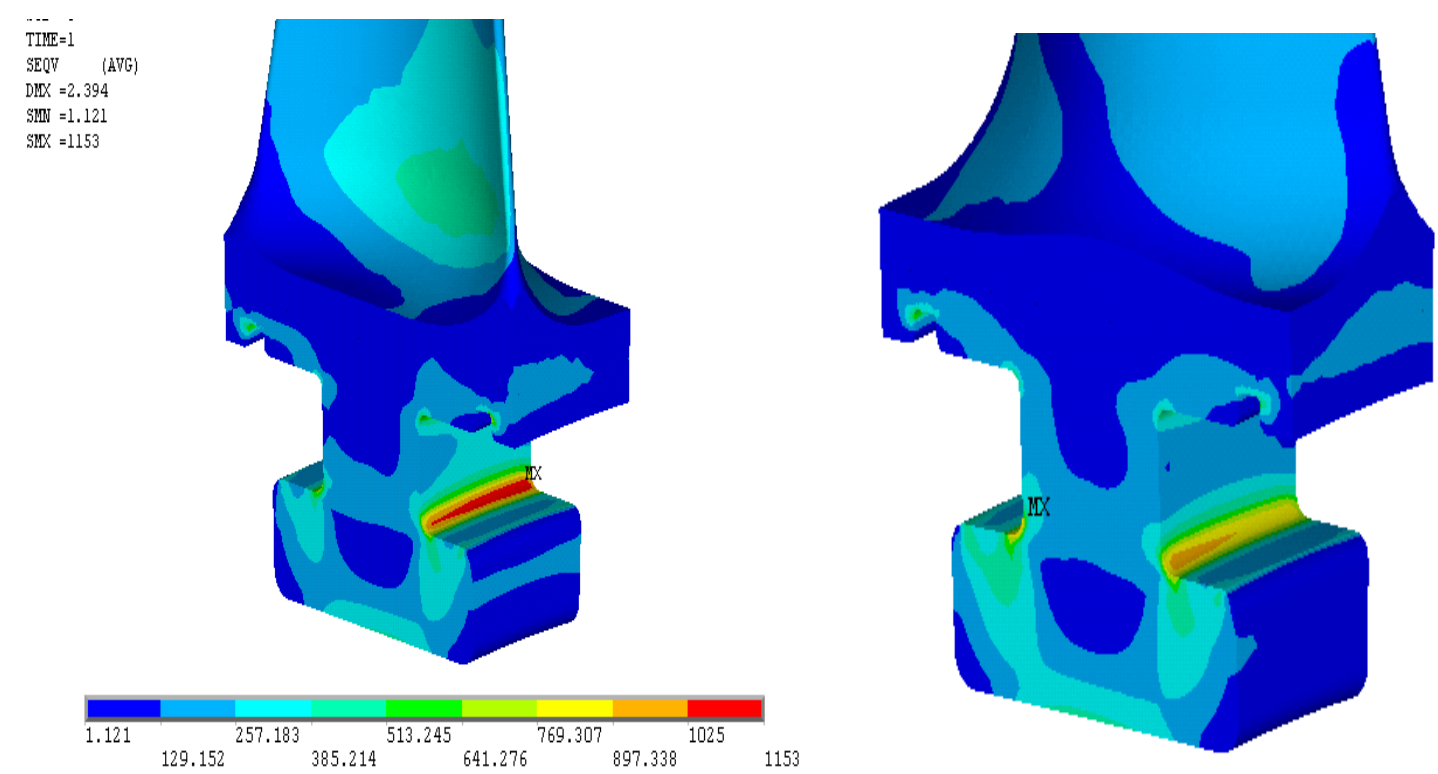

Figure 7: Von Mises stress in the elastic domain of the blade root at $6000 \mathrm{rpm}$

The following observations were made pertaining to the LP bladed disk assembly of steam turbine at its design speed of $6000 \mathrm{rpm}$ with appropriate boundary conditions as explained earlier. For the baseline model, a linear stress analysis approach has shown a peak stress of $1904 \mathrm{MPa}$ in the blade root fillet and $1660 \mathrm{MPa}$ at the disk 
hook fillet, which when Neuberised lead to a very poor LCF life of 2400 cycles. From a gross yielding point of view, the average stress in the blade neck is within the desired allowable limit. The blade root fillet section was strengthened by providing a generous fillet at the root. The modifications were made based on Peterson's SCF chart for the given $(r / d)$ ratio subjected to tensile loading. This modification successfully brought down the stress concentration to $1153 \mathrm{MPa}$ in the blade root fillet and the peak stress of $1102 \mathrm{MPa}$ is seen at the disk hook fillet. The corresponding values improved the LCF to 6200 cycles in the blade and 7100 cycles in the disk. The design rule developed is successfully utilized to provide the structural integrity in turbo machinery the blades and disk. These rules act as design checks for verifying the finite element analysis results at various design points such as the average section stress, over speed margin, and radial growth.

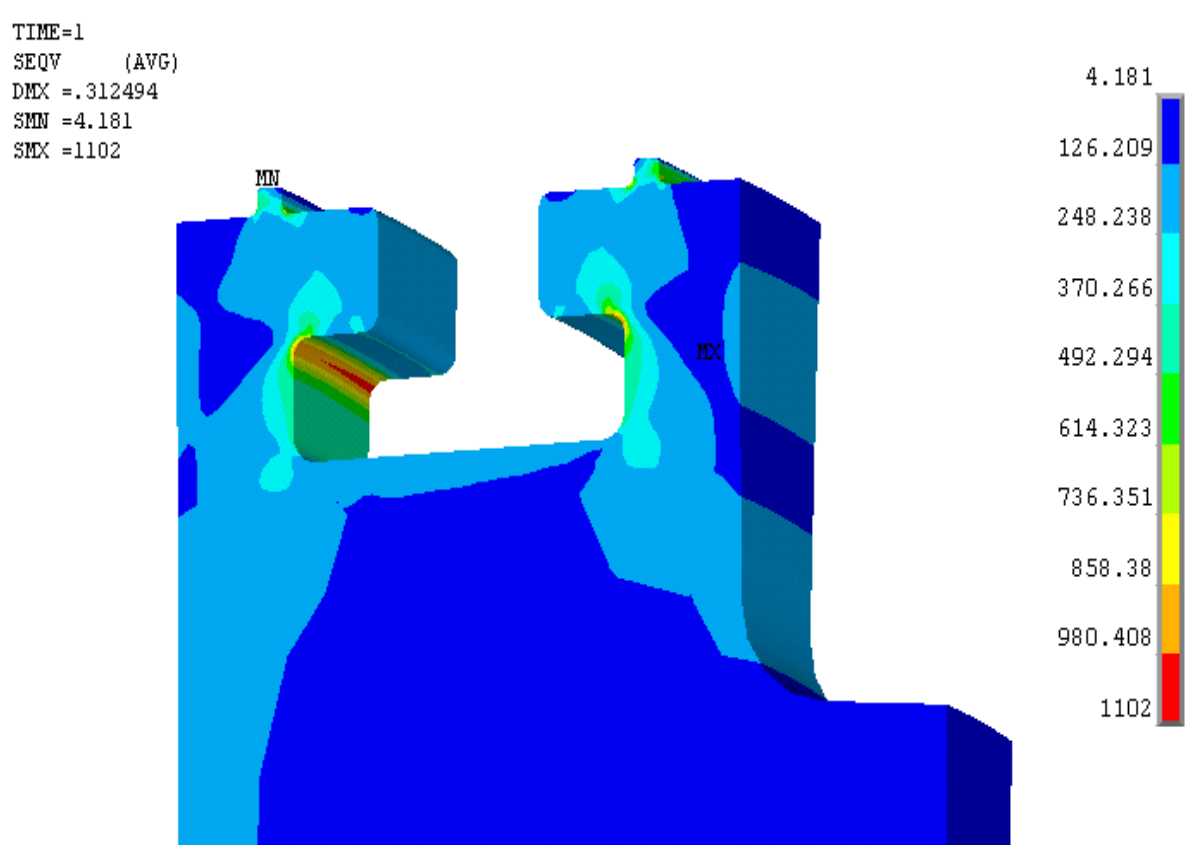

Figure 8: Von Mises stress in the elastic domain of the disk at $6000 \mathrm{rpm}$

\section{CONCLUSIONS}

The following conclusions can be drawn from the present work.

a) Custom made methodological procedure is used for strength evaluation of a LP steam turbine bladed disk assembly using FEA results and classical approach.

b) The developed design rule was successfully used in understanding and evaluating the structural requirements and design considerations to be incorporated during the design of turbo machinery components.

c) Based on Peterson's chart, the stress concentration factor was minimized and the local strain approach was used to achieve the required LCF cycle using Neuber's rule. 


\section{ACKNOWLEDGEMENT}

The authors are thankful to Dr. K Ramachandra, former director of the Gas Turbine Research Establishment, Bangalore, India, for his support.

\section{REFERENCES}

Bloch, H.P. (1982) A Practical Guide To Steam Turbine Technology. New York: McGraw Hill.

Kearton, W.J. (1988) Steam Turbine Theory And Practice. Seventh Edition. New Delhi: CBS Publishers.

Kim, H.J. (1998) Fatigue Failure Analysis Of Last Stage Blade In A Low Pressure Steam Turbine. Engineering Failure Analysis, 5: 93-100.

Kubiak Sz, J., Segura, J.A., Gonzalez R. G., García, J.C., Sierra E. F., Nebradt G.J., and Rodriguez, J.A. (2009) Failure Analysis of the 350 MW Steam Turbine Blade Root. Engineering Failure Analysis, 16(4): 1270-1281.

Mazur, Z., Illescas, R.G., Romano, J.A., and Rodriguez, N.P. (2008) Steam Turbine Blade Failure Analysis. Engineering Failure Analysis, 15(1-2): 129-14.

Mazur, Z., Illescas, R.G., and Calderón, J.P. (2009) Last Stage Blades Failure Analysis of a $28 \mathrm{MW}$ Geothermal Turbine. Journal of Engineering Failure Analysis, 16(4): 1020-1032.

Pilkey, W.D. (1997) Peterson's Stress Concentration Factors. New York: Wiley.

Richter, C.H. (2003) Structural Design Of Modern Steam Turbine Blades Using ADINA. Computers and Structures, 81: 919-927. 